\title{
Orthostatic hypertension: home blood pressure monitoring for detection and assessment of treatment with doxazosin
}

\author{
Satoshi Hoshide ${ }^{1,2}$, Gianfranco Parati $^{3}$, Yoshio Matsui ${ }^{1}$, Seiichi Shibazaki ${ }^{1}$, Kazuo Eguchi ${ }^{1}$ \\ and Kazuomi Kario ${ }^{1,2}$
}

To determine the role of home blood pressure (BP) monitoring for a reproducible assessment of orthostatic hypertension (OHT) and the effectiveness of hypertension control by doxazosin. In this study, 605 medicated hypertensive outpatients were enrolled. Home BP in the sitting and standing positions was monitored in all patients in the morning and evening for 6 months. According to an open-label multicenter trial design, the patients were randomly allocated to either an intervention group that took doxazosin (1-4 mg) at bedtime or to a control group that did not receive any add-on medication. The patients were divided into deciles of orthostatic BP change as evaluated by home BP monitoring at baseline. Those in the top decile, in the lowest decile and in deciles two through eight were then assigned to the $\mathrm{OHT}$ group, the orthostatic hypotension group and the orthostatic normotension group, respectively.Orthostatic BP in the OHYPO group did not change, whereas that of the OHT group was markedly reduced by doxazosin $(P<0.01)$. In the control group, classification into orthostatic BP categories using home BP monitoring was more reproducible ( $\kappa$ coefficient: $0.42-0.50$ ) than when using clinical BP ( $\kappa$ coefficient: $0.13-0.24$ ). In all groups, a reduction in the urinary albumin/creatinine ratio was significantly associated with a reduction in orthostatic BP doxazosin $(P<0.001)$. The identification of $\mathrm{OHT}$ based on home BP monitoring was highly reproducible. The administration of doxazosin might control $\mathrm{OHT}$ and consequently prevent target organ damage.

Hypertension Research (2012) 35, 100-106; doi:10.1038/hr.2011.156; published online 15 September 2011

Keywords: doxazosin; home blood pressure; orthostatic hypertension; urinary albumin

\section{INTRODUCTION}

Shifting from a sitting or the supine position to an upright posture is accompanied by either a reduction, no change or an increase in blood pressure (BP). ${ }^{1,2}$ The last condition, referred to as orthostatic hypertension (OHT), has been reported to be associated with target organ damage such as silent cerebral infarction, cardiac hypertrophy and urinary albuminuria. ${ }^{3-5}$

In most previous studies, post-ural BP changes have been evaluated by clinical BP or by BP readings taken during head up-tilt test, although the reproducibility of these BP measurements is still matter of controversy. ${ }^{3,5-8}$ Home BP monitoring (HBPM) has been recently recommended as a convenient and reproducible method of $\mathrm{BP}$ measurement in hypertension management, with a better prognostic value than clinical BP measurements. ${ }^{9-11}$ However, no data are available on the reproducibility of orthostatic BP changes when assessed by means of HBPM, which we previously showed to be more closely associated with increased albuminuria than when patients were assessed by clinical BP. ${ }^{4}$
Hyperactivation of the sympathetic nervous system has been suggested as one of the mechanisms of OHT, in line with our previous finding that BP increase during head-up tilt in OHT subjects was blunted by alpha-adrenergic blockade. ${ }^{5}$ However, alpha blockers are not commonly used in this context, because orthostatic hypotension (OHYPO) is known to be a frequent side effect of alpha-adrenergic blockade. Selective alpha-1 receptor blockade by doxazosin was reported to be less frequently associated with OHYPO than was administration of traditional alpha-adrenergic blockers. ${ }^{12}$ To date, no report is available on the ability of doxazosin to reduce the orthostatic BP rise in subjects with OHT and to counteract its negative effect in terms of organ damage.

Our study was aimed at filling these gaps in our current knowledge by investigating the reproducibility of orthostatic BP changes detected by HBPM and the association between the effects of doxazosin on orthostatic BP changes and on urinary albumin excretion in OHT patients.

${ }^{1}$ Division of Cardiovascular Medicine, Department of Medicine, Jichi Medical University School of Medicine, Tochigi, Japan; ${ }^{2}$ Department of Sleep and Circadian Cardiology, Jichi Medical University School of Medicine, Tochigi, Japan and ${ }^{3}$ Department of Clinical Medicine and Prevention, University of Milano-Bicocca, Milan, Italy

Correspondence: Dr S Hoshide, Department of Medicine, Division of Cardiovascular Medicine, Jichi Medical University School of Medicine, 3311-1, Yakushiji, Shimotsuke-city 329-0434, Japan.

E-mail: hoshide@jichi.ac.jp

Received 6 May 2011; revised 24 June 2011; accepted 28 June 2011; published online 15 September 2011 


\section{METHODS}

Our study focused on home BP data obtained while assessing orthostatic BP changes in patients in the Japan Morning Surge (JMS) 1 Study. ${ }^{13}$ The study was conducted from 1 August 2003 to the end of August 2005 by 20 doctors at 16 institutions in Japan. The Ethics Committee of the Internal Review Board at Jichi Medical University (Tochigi, Japan) approved the protocol. Written, informed consent was obtained from each subject enrolled in this study.

\section{Study subjects and study design}

We enrolled in our study 605 hypertensive subjects older than 18 years who had received stable antihypertensive treatment (with the exclusion of alpha-blockers or beta-blockers) for 3 months and whose morning BP levels measured by HBPM were $\geqslant 135 / 85 \mathrm{~mm} \mathrm{Hg}$. We did not recruit patients with arrhythmias, history of heart failure, OHYPO (a drop in BP of $20 \mathrm{~mm} \mathrm{Hg}$ in systolic BP (SBP) or $10 \mathrm{~mm} \mathrm{Hg}$ in diastolic BP from sitting to standing) with symptoms, dementia, malignancy or chronic inflammatory disease and those defined at high risk according to the current Japanese Hypertension Guidelines at the time. ${ }^{14}$ The enrolled subjects were randomized to a control group that received no alteration of the previous treatment regimen and only reinforcement of lifestyle changes during follow-up, or to an intervention group that received doxazosin $(1 \mathrm{mg})$ once daily at bedtime, in addition to the previous treatment regimen. The dose of doxazosin was increased to $2 \mathrm{mg}$ per day and then to $4 \mathrm{mg}$ per day at follow-up visits performed at monthly intervals until morning SBP was $<135 \mathrm{~mm} \mathrm{Hg}$. If morning SBP was still $\geqslant 135 \mathrm{~mm} \mathrm{Hg}$ after $4 \mathrm{mg}$ per day of doxazosin, then a beta-blocker (atenolol, $25 \mathrm{mg}$ per day) was added once daily at bedtime. Randomization was carried out at an independent research center through a computerized system. The follow-up study period was 6 months. Treatment with the other baseline antihypertensive medication remained unchanged throughout this study period. There was no significant difference in the frequency of major events between the two groups. The details regarding major events in this series have been reported previously. ${ }^{15}$

\section{BP measurements}

Methods for BP measurement of patients in the sitting and standing positions in the clinic and at home have been described previously. ${ }^{4}$ Clinical BP was measured by a validated oscillometric device (HEM-705IT, Omron Healthcare, Kyoto, Japan), by considering the mean of two consecutive measurements in a patient in the sitting position and the mean of another two measurements with the patient in the standing position. Patients were instructed to place the cuff on the same arm for all measurements, and sitting BP was measured after more than 2 min of seated rest, twice per occasion, with a 15-s interval between the measurements. The subject then stood up for a 30-s interval after which standing BP was measured, again twice per occasion, with a 15-s interval between the measurements. The total standing period was approximately $2 \mathrm{~min}$.

At home, morning $\mathrm{BP}$ and evening $\mathrm{BP}$ were measured using the same device (HEM-705IT) at that used in the clinic, for a period of 3 days, in accordance with the Japanese Society of Hypertension Guidelines. ${ }^{16}$ The measurements obtained while the patient was in the standing position were performed in the same manner at home and in the clinic.

The HBPM values were obtained by averaging two measurements in the morning and two measurements in the evening for each of 3 HBPM days (12 readings total) with the patient in the sitting position, and the mean was calculated for the same number of readings taken while the patient was standing. We assessed the orthostatic BP change, measured by HBPM, as the mean of standing SBP (morning and evening, 12 readings total) minus the mean of sitting SBP (also morning and evening; 12 readings total). At baseline, we classified all patients as follows, that is, according to the extent of orthostatic BP change, and with distinct classifications based on clinic and home measurements: OHT (OHT): patients in the top decile of orthostatic BP change; OHYPO: patients in the lowest decile of orthostatic BP change; and orthostatic normotension (ONT): patients with intermediate orthostatic BP changes.

\section{Blood and urine samples}

Blood and urine samples were collected in the morning, with subjects fasting, at the beginning of the study and at the end of the 6-month followup period. Urinary albumin was measured using the immunoturbidimetric method (Mitsubishi Chemical Iatron, Tokyo, Japan), and expressed as the urinary albumin/creatinine ratio (UAR, $\mathrm{mg} / \mathrm{gCr}$ ). The UAR was calculated from a morning spot-urine sample. Both serum and urine creatinine were measured by the Jaffe reaction without deproteinization and then the results were quantified by a photometric method. All assays were performed at the Mitsubishi Biochemical Laboratory, and the intra- and inter-coefficients of variation were 1.52 and $2.48 \%$, respectively, for the urinary albumin assay.

\section{Statistical analysis}

All primary analyses were performed on an intention-to-treat basis. Data are expressed as means ( \pm s.d.) or percentages. As the distributions of UAR were highly skewed, these parameters were log-transformed before statistical analysis. One-way analysis of variance (ANOVA) was performed to detect differences among groups, and Tukey's honestly significant difference test was used for multiple pairwise comparisons of means among groups. The $\chi^{2}$-test was used to evaluate differences in prevalence rates. The degree of concordance of the orthostatic BP categories defined on the basis of clinic or home BP measurements throughout follow-up was evaluated using the $\kappa$ coefficient. Significant differences between the control and intervention group were used to investigate the time course of BP values by repeated-measures ANOVA; then, post-hoc comparisons between the control and intervention groups were made using the Bonferroni comparison procedure. Multiple linear regression analysis was performed to estimate and test the independent effects of $\mathrm{BP}$ and of doxazosin administration on UAR adjusting for age, sex, body mass index, administration of doxazosin, the use of beta-blocker, sitting SBP at HBPM and UAR at baseline. In a two-sided test within each arm of the study, 50 patients in the OHT group were required to detect a mean difference in orthostatic BP change of $13 \mathrm{~mm} \mathrm{Hg}$ (s.d., $15 \mathrm{~mm} \mathrm{Hg}$ ) between the doxazosin treatment and control groups ( $80 \%$ power and $5 \%$ significance). Differences with a $P$-value of $<0.05$ (two-tailed) were considered statistically significant throughout the study. The computer software package SPSS version 11.0J (SPSS, Chicago, IL, USA) was used for the analyses.

\section{RESULTS}

Table 1 shows the baseline characteristics of all patients divided into groups according to the orthostatic BP changes determined by HBPM.

\section{Changes during follow-up in sitting BP and orthostatic BP as determined by HBPM or clinical BP monitoring}

As shown in Figure 1, as regards the HBPM data, repeated-measures ANOVA showed that there was a significant difference between the control and intervention groups from 0 to 6 months in both the OHYPO and ONT groups. In the OHYPO group, subjects receiving doxazosin displayed a significantly lower sitting SBP at 3 and 6 months of follow-up than subjects in the control group. Similarly, in the ONT group, sitting SBP was significantly lower at 2-6 months of follow-up in the intervention group than in the control group, but in the OHT group, there were no significant differences in sitting SBP between control and intervention groups during follow-up. As regards clinical BP measurement, the repeated-measures ANOVA revealed a significant difference between the control and intervention groups from 0 to 6 months in the ONT group, and in the post-hoc test, sitting SBP was significantly lower at 1-6 months of follow-up in the intervention group than in the control group; however, again in the OHT group, no significant difference in sitting SBP was noted during the follow-up between the control and intervention groups.

As regards orthostatic SBP changes (Figure 2) observed by HBPM of the OHYPO and ONT groups, repeated-measures ANOVA showed no significant difference between the control and intervention groups 


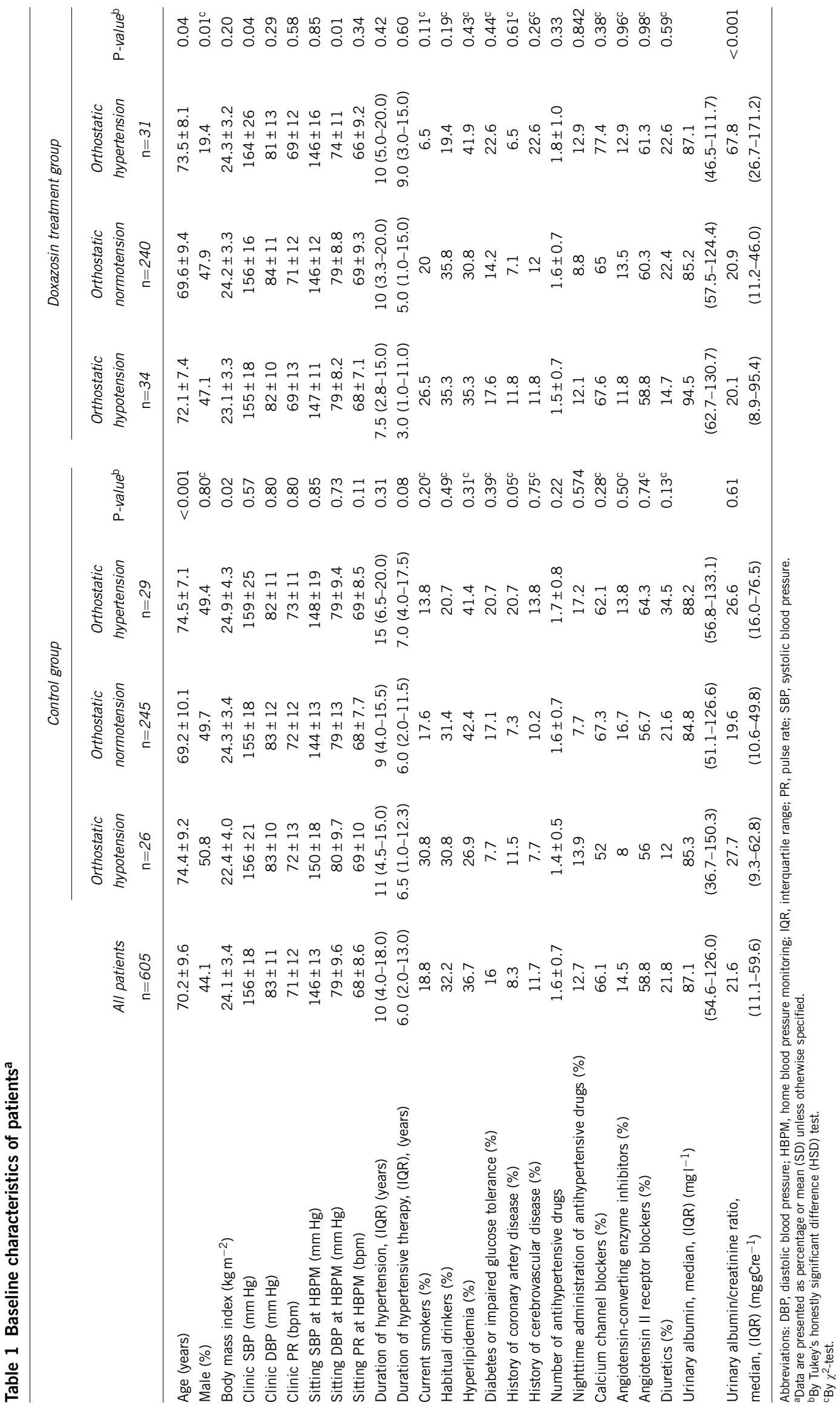




\section{At home}

Orthostatic hypotension group

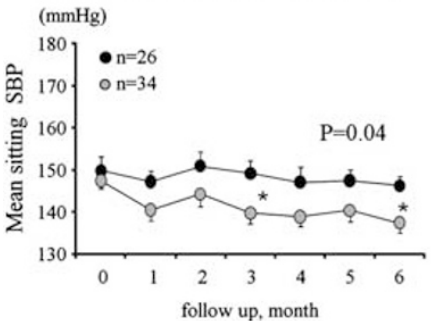

Orthostatic hypotension group

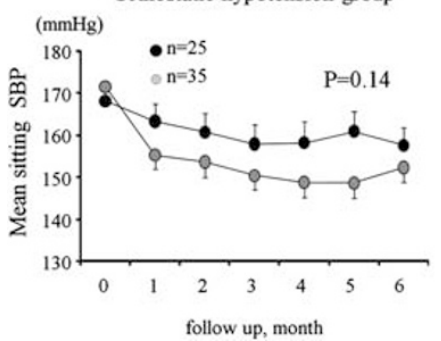

Orthostatic normotension group

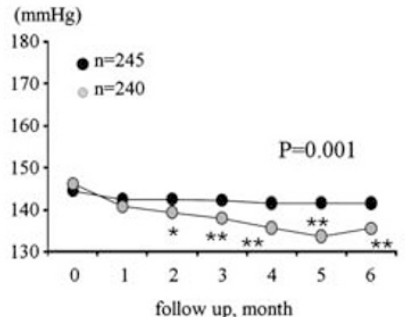

At clinic

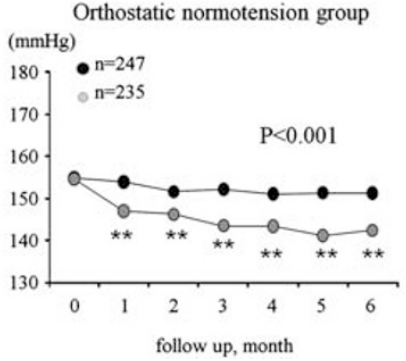

Orthostatic hypertension group

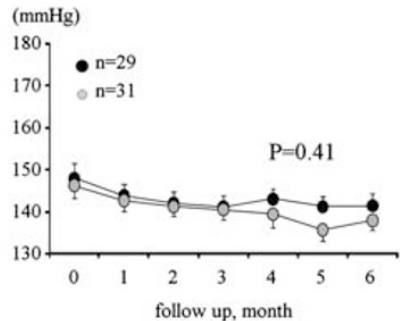

Orthostatic hypertension group

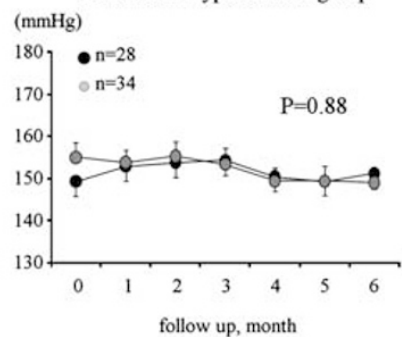

Figure 1 Mean sitting systolic BP (SBP) at home and in the clinic in control and intervention groups. The BP (BP) values and SEs shown in the upper figure (at-home measurement) are data for orthostatic BP changes determined by HBPM, and those in the lower figure (in-clinic measurement) are data for orthostatic BP changes determined by BP measured in the clinic. Black circles and gray circles indicate the control group and intervention group, respectively. Error bars indicate SEs. $P$-values refer to comparison between the two groups (repeated-measures ANOVA). Asterisks indicate differences between the control and intervention groups at the same follow-up month, determined by post-hoc test. ${ }^{*} P<0.05,{ }^{* *} P \leqslant 0.001$.

At home
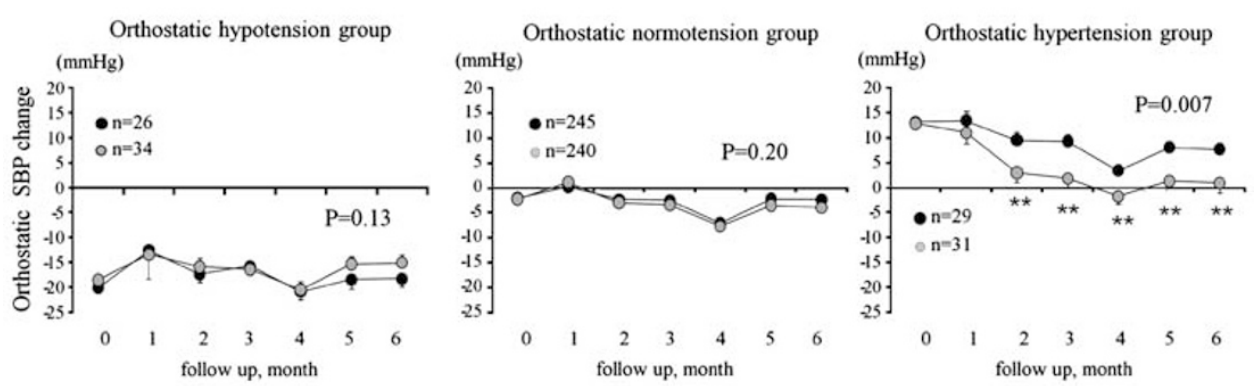

At clinic
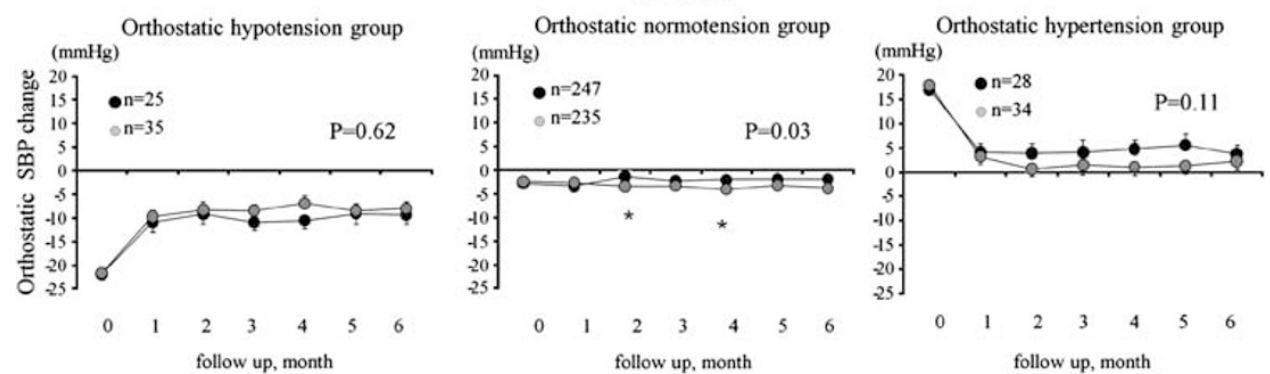

Figure 2 Mean orthostatic systolic BP (SBP) changes at home and in the clinic in control and intervention groups. The BP (BP) values and SEs shown in the upper figure (at-home measurement) are data for orthostatic BP changes determined by HBPM, and those in the lower figure (in-clinic measurement) are data for orthostatic BP changes determined based on in-clinic BP measurement. Black circles and gray circles indicate control group and intervention group, respectively. Error bars indicate SEs. $P$-values refer to comparisons between the two groups (repeated-measures ANOVA). Asterisks indicate differences between the control and intervention groups at the same follow-up month, determined by post-hoc test. ${ }^{*} P<0.05,{ }^{* *} P \leqslant 0.01$. 
in terms of the SBP reduction observed under the standing condition. In addition, repeated-measures ANOVA showed a significant difference between control and intervention OHT groups during follow-up, namely, there was a significantly less-pronounced SBP increase in patients treated with doxazosin than in those in the control group at 2-6 months of follow-up, according to the post-hoc test. In the ONT group, there was a significant difference between the control and intervention groups' clinical BP measurements, that is, subjects receiving doxazosin displayed a significantly lower standing SBP at 2 and 4 months of follow-up than did subjects in the control group. However, in the OHYPO group, there was no significant difference between control and intervention groups in terms of the orthostatic SBP reduction throughout the follow-up period. Finally, in the OHT group categorized using clinical BP monitoring, the orthostatic SBP increase was not significantly different between the doxazosin-treated and control groups.

When we used the category of OHT evaluated by HBPM at baseline and at 6 months, the prevalence of OHT changed from 9.7\% (29/300) at baseline to $11.0 \%(33 / 300)$ at 6 months in the control group. In the intervention group, that prevalence changed from $10.2 \%(31 / 305)$ at baseline to $5.2 \%(16 / 305)$ at 6 months $(P=0.02)$.

\section{Reproducibility of subjects' classification in each orthostatic} BP category among the controls

The reproducibility of subjects' classification in each orthostatic BP category, defined by HBPM and clinical BP throughout the follow-up period, is shown in Table 2. The concordance in the definition of the three orthostatic BP categories using HBPM was consistently $>0.40$

Table 2 Kappa coefficient of orthostatic blood pressure change categories determined from home blood pressure and clinic blood pressure at baseline and monthly follow-up

\begin{tabular}{lcc}
\hline & Using home blood pressure & Using clinic blood pressure \\
\hline 1 month & 0.50 & 0.13 \\
2 month & 0.48 & 0.23 \\
3 month & 0.51 & 0.17 \\
4 month & 0.42 & 0.24 \\
5 month & 0.43 & 0.24 \\
6 month & 0.43 & 0.18 \\
\hline
\end{tabular}

( $\kappa$ coefficient) throughout the entire follow-up period, indicating good reproducibility, ${ }^{17}$ while the concordance in the corresponding definitions obtained by clinical BP remained $<0.40$ throughout the follow-up period.

Association between changes in UAR and changes in both sitting and standing BP during follow-up

The possible determinants of changes in UAR during follow-up were assessed through a multiple linear regression analysis model, including BP parameters obtained by HBPM (Table 3). The reduction in UAR during follow-up was significantly and independently associated with the administration of doxazosin, baseline-sitting SBP at HBPM, reduction in sitting SBP at HBPM and reduction in orthostatic SBP increases at HBPM during follow-up (Table 3). When clinical rather than home BP was included in a multiple regression analysis model, the reduction in UAR during follow-up was significantly and independently associated with the administration of doxazosin and with a reduction in sitting SBP measured in the clinic, while no significant association between reduction in UAR and reduction in orthostatic SBP increases at clinical visits was found (Table 3). Finally, by including both HBPM and clinical BP parameters in a more complex multiple regression analysis model, the reduction in UAR was significantly and independently associated with the reduction in sitting SBP determined by HBPM $(\beta \quad(95 \%$ CI $)=0.01 \quad(0.007-0.011)$, $P<0.001)$ and with the reduction in orthostatic SBP increases ( $\beta=0.04$ (0.001-0.007), $P=0.009$ ) determined by HBPM during the follow-up. Conversely, no association between reduction in UAR and reduction in sitting clinical SBP $(\beta=0.001$ (from -0.001 to 0.002 ), $P=0.32$ ) or reduction in orthostatic SBP increases at clinical visits ( $\beta=0.001$ (from -0.002 to 0.002 ), $P=0.66$ ) was found. There was no difference in the prevalence of microalbuminuria between baseline and 6-month follow-up in the control group ( $37.0 \mathrm{vs.} 36.7 \%, P=0.50)$, while the difference was significant in the intervention group (40.7 vs. $28.4 \%, P=0.001)$.

\section{DISCUSSION}

The initial novel result of note in the present study was that the reproducibility of the orthostatic SBP changes assessed by HBPM was significantly better than the reproducibility of the corresponding changes assessed by clinical BP measurements, because the $\kappa$ coefficient value of orthostatic SBP changes assessed by HBM indicated a range from 0.42 to 0.51 , that is, moderate agreement for orthostatic

Table 3 Multiple linear regression analysis of orthostatic systolic BP change at HBPM and clinic for reduction of urinary albumin/creatinine ratio in the entire group $(n=605)$

\begin{tabular}{|c|c|c|c|c|}
\hline & \multicolumn{2}{|l|}{$H B P M$} & \multicolumn{2}{|l|}{ Clinic } \\
\hline & Non-standardized B coefficient $(95 \% \mathrm{Cl})$ & P-value & Non-standardized B coefficient $(95 \%$ Cl) & P-value \\
\hline Age & -0.002 (from -0.004 to 0.001 ) & 0.230 & 0.001 (from -0.004 to 0.002 ) & 0.486 \\
\hline Sex & 0.020 (from -0.028 to 0.066 ) & 0.418 & 0.022 (from -0.027 to 0.072 & 0.364 \\
\hline Body mass index & 0.001 (from -0.007 to 0.007 ) & 0.986 & 0.002 (from -0.005 to 0.010 ) & 0.534 \\
\hline Administration of beta blocker & -0.053 (from -0.120 to 0.012 ) & 0.110 & -0.029 (from -0.098 to 0.039 ) & 0.401 \\
\hline Reduction of orthostatic systolic BP change & 0.004 (from 0.001 to 0.007 ) & 0.005 & 0.001 (from -0.001 to 0.003 ) & 0.266 \\
\hline Reduction of sitting systolic BP & 0.010 (from 0.007 to 0.012 ) & $<0.001$ & 0.003 (from 0.002 to 0.005 ) & $<0.001$ \\
\hline Administration of doxazosin & -0.059 (from -0.119 to -0.000 ) & 0.050 & -0.113 (from -0.175 to -0.051 ) & $<0.001$ \\
\hline Sitting systolic baseline BP & $\begin{array}{c}0.006 \text { (from } 0.004 \text { to } 0.008 \text { ) } \\
\qquad R^{2}=0.24\end{array}$ & $<0.001$ & $\begin{array}{c}0.002 \text { (from } 0.001 \text { to } 0.004 \text { ) } \\
\qquad R^{2}=0.15\end{array}$ & 0.004 \\
\hline
\end{tabular}

Abbreviations: BP, blood pressure; $\mathrm{Cl}$, confidence interval; HBPM, home blood pressure monitoring. 
BP categories, while BP assessed in the clinic showed a $\kappa$ coefficient range of $0.13-0.24$, which indicates slight or fair agreement. ${ }^{17}$ The measurement of BP in a clinical setting tends to be a stressor itself, and can falsely increase BP and BP reactivity through what is recognized as the white coat effect. ${ }^{18}$ Our observations provide additional support for the clinical usefulness of HBPM, ${ }^{19}$ as compared with that of conventional clinical BP readings, ${ }^{9-11}$ by demonstrating that HBPM data are likely to show good reproducibility, not only for sitting BP levels but also with respect to orthostatic BP changes.

A second important result of the present study was that the administration of doxazosin significantly ameliorated the increase in SBP while standing observed in treated hypertensive patients with OHT. In contrast, doxazosin did not enhance the relative decrease in SBP measured in standing OHYPO patients. Finally, as regards the HBPM data, the decrease in orthostatic SBP in both the control and doxazosin-treated groups was associated with a decrease in UAR, independently of a concomitant decrease in sitting SBP level.

Orthostatic BP changes may depend on several factors and may reflect underlying alterations in cardiovascular control mechanisms. OHYPO is commonly caused as a result of either age-related changes in BP-regulatory mechanisms or hypertensive drug therapy. ${ }^{20}$ Because we enrolled elderly hypertensive patients on medication in this study, OHYPO might be observed. On the other hand, OHT might be part of the BP hyper-reactivity pattern often observed in hypertensive patients, independently contributing to the risk of cardiovascular complications. Our findings regarding doxazosin administration provide new information about the mechanisms underlying orthostatic BP changes, namely, treatment with doxazosin was found to significantly ameliorate the standing SBP increase in the OHT group throughout the follow-up period. In a previous report, we had already observed a suppression of orthostatic SBP increase in elderly hypertensives with OHT following doxazosin administration. ${ }^{5}$ However, in that previous study, we evaluated orthostatic SBP once before and once after the administration of doxazosin only. In contrast, in the present study, we included several observations obtained over a 6month follow-up period, and it was found that the continuous administration of doxazosin successfully reduced not only sitting SBP but also the SBP increase while standing observed in OHT subjects throughout the follow-up period; furthermore, this amelioration of symptoms was seen with both clinic and home BP-monitoring modalities.

It is known that UAR is an important surrogate marker of cardiovascular risk, as the UAR has been reported to be associated with cardiovascular events in both diabetic and non-diabetic hypertensive patients. ${ }^{21,22}$ An additional interesting finding of the present study was that the administration of doxazosin not only successfully diminished orthostatic SBP changes, but it also reduced renal organ damage, as quantified by decreased UAR. Moreover, this reduction in UAR was related to a reduction in orthostatic SBP increases in a manner independent of the decrease in sitting SBP. In addition, the UAR reduction was better predicted using HBPM than clinical BP measurements.

Although many previous studies have reported an association of UAR with either sitting BP levels in the clinic or at home and 24-h or nighttime ambulatory BP levels, ${ }^{15,23,24}$ our report is the first to demonstrate a relationship between a reduction in UAR and the pharmacological reduction of OHT via a drug antagonist of the sympathetic nervous system. The mechanism by which the suppression of orthostatic BP increase led to a reduction of UAR in our study remains to be investigated in greater detail. However, it is possible that such a reduction could be due to the administration of a drug that antagonizes sympathetic activity in the kidneys. It is known that the sympathetic nervous system is overactive in patients with chronic renal damage. ${ }^{25}$ Amman et al. ${ }^{26}$ reported that alpha- and beta-blockers, and their combination, had a beneficial effect on renal morphology and albuminuria in a rat model of renal ablation. Moreover, it has been reported that OHT might be associated with overactivity of the sympathetic nervous system. ${ }^{1,5,27}$ Therefore, doxazosin might break the vicious cycle between the sympathetic nervous hyperactivity, orthostatic BP increase and renal damage by reducing both the orthostatic BP increase and excessive urinary albumin excretion.

The blockade of alpha adrenergic receptors did not appear to aggravate the orthostatic BP decrease found in OHYPO subjects. The reason may be partly explained by the dosing time of doxazosin. The maximum reduction in supine and standing BP usually occurs between 2 and $6 \mathrm{~h}$ after administration of doxazosin at bedtime. ${ }^{28}$ Therefore, the bedtime administration may avoid excessive orthostatic $\mathrm{BP}$ reduction during daytime. In addition, the size of the OHYPO group may have been too small to detect differences in changes in orthostatic BP between the control and intervention groups. The lack of a significant difference between sitting BP values in the OHT group may have been related to the same issue regarding the sample size. There were some limitations in this study. We did not use a doubleblind study design. In addition, analysis of the JMS-1 data reported in this paper was not the primary focus of the study. Therefore, we did not randomize subjects for this study in particular. Finally, our results did not demonstrate that doxazosin is superior to any other antihypertensive drug for achieving reductions in orthostatic BP change and UAR.

Our results may challenge the recent tendency to avoid the use of an alpha-adrenergic-blocking drug as a first-line antihypertensive treatment, a tendency that was originated by publication of the results of the Antihypertensive and Lipid-Lowering treatment to prevent Heart Attack Trial trial, in which the doxazosin limb of the study was prematurely terminated because, while in this limb there was a similar rate of fatal cardiovascular events as in the chlortalidone limb, stroke and heart failure occurred more often. ${ }^{29}$ However, in the Anglo-Scandinavian Cardiac Outcomes Trial-BP Lowering Arm, the use of doxazosin as a third-line antihypertensive agent was not associated with an excess of cardiovascular events, and in particular of heart failure. ${ }^{30}$

In this study, we used active standing test (from the sitting to the standing position) for the evaluation of orthostatic BP change. Although the head-up tilt test is the gold standard method for the evaluation of post-ural BP changes, it is difficult to use the head-up tilt test routinely in clinical practice because special equipment is needed, and the procedure is time consuming. The change in $\mathrm{BP}$ on active standing test is recommended in the Japanese Society of Hypertension Guideline, ${ }^{9}$ although previous studies have used the definition of orthostatic BP change based on the change in BP from the supine to the standing position. ${ }^{6-8}$ In summary, the present study revealed that orthostatic BP changes detected by HBPM had better reproducibility than the corresponding BP changes detected by clinic BP measurements, and a closer correlation with UAR was obtained with HBPM. In the group of patients with OHT, treatment with doxazosin suppressed the orthostatic BP increase, which in turn might have reduced the UAR. Our results provide further support for the clinical usefulness of HBPM, in particular with regard to diagnosing patients with OHT, as well as in the context of clinical intervention. 


\section{CONFLICT OF INTEREST}

The authors declare no conflict of interest

\section{ACKNOWLEDGEMENTS}

The present study was financially supported, in part, by a grant from the Japan Heart Foundation, Tokyo. Participants and participating centers: Shizukiyo Ishikawa: Jichi Medical University School of Medicine; Kazuo Eguchi: Jichi Medical University School of Medicine, Shioya General Hospital and Sano Municipal Hospital; Toru Hashimoto: Jichi Medical University School of Medicine; Masato Morinari: Jichi Medical University School of Medicine; Satoshi Hoshide: Jichi Medical University School of Medicine and Oyama Municipal Hospital; Yoko Hoshide: Sato Clinic; Motoyuki Ishiguro: Ishiguro Clinic; Toshio Nakayama: Nakayama Clinic; Hideo Hirose: Kosaka-cho Clinic; Naoshi Yamada: Yamada Brain Surgery Clinic; Akira Yoshimura and Makoto Yamashita: Mishima Clinic; Masanori Harada and Hitoshi Nishimura: Nishiki Central Hospital; Ruri Kaneda: Jyosai Hospital; Yoshio Matsui and Seiichi Shibasaki: Miwa Municipal Hospital; Mitsunobu Murata: Koga Red Cross Hospital; Joji Ishikawa: Koga Red Cross Hospital and Sano Municipal Hospital; and Yasuyuki Mizumori: Ieshima Clinic and Uzuka Clinic (20 physicians and 16 institutes). The coordinating committees were Yumiko Yoshida, Tsutomu Sudo, and Kazuo Okuhara from Biomedis, Safety committee: Yoshiaki Murakami, Takeshi Mitsuhashi, Takaaki Katsuki: Jichi Medical University School of Medicine. Dr Kario was supported by a grant from the Japan Heart Foundation (JHF-03-143). http://ClinicalTrials.gov: \#NCT00285519, UMIN (University Hospital Medical Information Network) Clinical Trials Registry (UMIN-CTR): \#C000000309.

1 Fessel J, Robertson D. Orthostatic hypertension: when pressor reflexes overcompensate. Nat Clin Pract Nephrol 2006; 2: 424-431.

2 Lipsitz LA. Orthostatic hypotension in the elderly. N Eng/ J Med 1989; 321: 952-957.

3 Eguchi K, Kario K, Hoshide S, Hoshide Y, Ishikawa J, Morinari M, Hashimoto T, Shimada K. Greater change of orthostatic blood pressure is related to silent cerebral infarct and cardiac overload in hypertensive subjects. Hypertens Res 2004; 27 : 235-241.

4 Hoshide S, Matsui Y, Shibasaki S, Eguchi K, Ishikawa J, Ishikawa S, Kabutoya T, Schwartz JE, Pickering TG, Shimada K, Kario K. Orthostatic hypertension detected by self-measured home blood pressure monitoring: a new cardiovascular risk factor for elderly hypertensives. Hypertens Res 2008; 31: 1509-1516.

5 Kario K, Eguchi K, Hoshide S, Hoshide Y, Umeda Y, Mitsuhashi T, Shimada K. U-curve relationship between orthostatic blood pressure change and silent cerebrovascular disease in elderly hypertensives: orthostatic hypertension as a new cardiovascular risk factor. J Am Coll Cardiol 2002; 40: 133-141.

6 Eigenbrodt ML, Rose KM, Couper DJ, Arnett DK, Smith R, Jones D. Orthostatic hypotension as a risk factor for stroke: the atherosclerosis risk in communities (ARIC) study, 1987-1996. Stroke 2000; 31: 2307-2313.

7 Matsubayashi K, Okumiya K, Wada T, Osaki Y, Fujisawa M, Doi Y, Ozawa T. Postural dysregulation in systolic blood pressure is associated with worsened scoring on neurobehavioral function tests and leukoaraiosis in the older elderly living in a community. Stroke 1997; 28: 2169-2173.

8 Masaki KH, Schatz IJ, Burchfiel CM, Sharp DS, Chiu D, Foley D, Curb JD. Orthostatic hypotension predicts mortality in elderly men: the Honolulu heart program. Circulation 1998; 98: 2290-2295.

9 Ogihara T, Kikuchi K, Matsuoka H, Fujita T, Higaki J, Horiuchi M, Imai Y, Imaizumi T, Ito S, Iwao H, Kario K, Kawano Y, Kim-Mitsuyama S, Kimura G, Matsubara H, Matsuura H, Naruse M, Saito I, Shimada K, Shimamoto K, Suzuki H, Takishita S, Tanahashi N, Tsuchihashi T, Uchiyama M, Ueda S, Ueshima H, Umemura S, Ishimitsu T, Rakugi H.
The Japanese society of hypertension guidelines for the management of hypertension (JSH 2009). Hypertens Res 2009; 32: 3-107.

10 Bobrie G, Chatellier G, Genes N, Clerson P, Vaur L, Vaisse B, Menard J, Mallion J-M. Cardiovascular prognosis of "Masked hypertension" detected by blood pressure self-measurement in elderly treated hypertensive patients. JAMA 2004; 291: 1342-1349.

11 Little P, Barnett J, Barnsley L, Marjoram J, Fitzgerald-Barron A, Mant D. Comparison of agreement between different measures of blood pressure in primary care and daytime ambulatory blood pressure. BMJ 2002; 325: 254.

12 Isso JL, Sica DA, Black HR. Hypertension Primer, 4th edn. Lippincott Williams \& Wilkins: Philadelphia, 2008, pp 450-452.

13 Ishikawa J, Hoshide S, Shibasaki S, Matsui Y, Kabutoya T, Eguchi K, Ishikawa S, Pickering TG, Shimada K, Kario K. The Japan Morning Surge-1 (JMS-1) study: protocol description. Hypertens Res 2006; 29: 153-159.

14 Japanese Society of Hypertension Guidelines Subcommittee for the Management of Hypertension. Guidelines for the management of hypertension for general practitioners. Hypertens Res 2001; 24: 613-634.

15 Kario K, Matsui Y, Shibasaki S, Eguchi K, Ishikawa J, Hoshide S, Ishikawa S, Kabutoya T, Schwartz JE, Pickering TG, Shimada K. An alpha-adrenergic blocker titrated by selfmeasured blood pressure recordings lowered blood pressure and microalbuminuria in patients with morning hypertension: The Japan Morning Surge-1 study. J Hypertens 2008; 26: 1257-1265.

16 Imai Y, Otsuka K, Kawano Y, Shimada K, Hayashi H, Tochikubo O, Miyakawa M, Fukiyama K. Japanese society of hypertension (JSH) guidelines for self-monitoring of blood pressure at home. Hypertens Res 2003; 26: 771-782.

17 Landis JR, Koch GG. The measurement of observer agreement for categorical data. Biometrics 1977; 33: 159-174.

18 Mancia G, Bertinieri G, Grassi G, Parati G, Pomidossi G, Ferrari A, Gregorini L, Zanchetti $A$. Effects of blood-pressure measurement by the doctor on patient's blood pressure and heart rate. Lancet 1983; 2: 695-698.

19 Parati G, Stergiou GS, Asmar R, Bilo G, de Leeuw P, Imai Y, Kario K, Lurbe E, Manolis A, Mengden T, O'Brien E, Ohkubo T, Padfield P, Palatini P, Pickering T, Redon J, Revera M, Ruilope LM, Shennan A, Staessen JA, Tisler A, Waeber B, Zanchetti A, Mancia G. European society of hypertension guidelines for blood pressure monitoring at home: a summary report of the second international consensus conference on home blood pressure monitoring. J Hypertens 2008; 26: 1505-1526.

20 Ward C, Kenny RA. Reproducibility of orthostatic hypotension in symptomatic elderly. Am J Med 1996; 100: 418-422.

21 Mogensen CE. Microalbuminuria predicts clinical proteinuria and early mortality in maturity-onset diabetes. N Engl J Med 1984; 310: 356-360.

22 Yudkin JS, Forrest RD, Jackson CA. Microalbuminuria as predictor of vascular disease in non-diabetic subjects. Islington diabetes survey. Lancet 1988; 2: 530-533.

23 Gaborieau V, Delarche N, Gosse P. Ambulatory blood pressure monitoring versus selfmeasurement of blood pressure at home: correlation with target organ damage. J Hypertens 2008; 26: 1919-1927.

24 Stergiou GS, Argyraki KK, Moyssakis I, Mastorantonakis SE, Achimastos AD, Karamanos VG, Roussias LG. Home blood pressure is as reliable as ambulatory blood pressure in predicting target-organ damage in hypertension. Am J Hypertens 2007; 20: 616-621.

25 Neumann J, Ligtenberg G, Klein II, Koomans HA, Blankestijn PJ. Sympathetic hyperactivity in chronic kidney disease: pathogenesis, clinical relevance, and treatment. Kidney Int 2004; 65: 1568-1576.

26 Amann K, Koch A, Hofstetter J, Gross ML, Haas C, Orth SR, Ehmke H, Rump LC, Ritz E. Glomerulosclerosis and progression: effect of subantihypertensive doses of alpha and beta blockers. Kidney Int 2001; 60: 1309-1323.

27 Yoshinari M, Wakisaka M, Nakamura U, Yoshioka M, Uchizono Y, Iwase M. Orthostatic hypertension in patients with type 2 diabetes. Diabetes Care 2001; 24: 1783-1786. 28 Taylor SH. Clinical pharmacotherapeutics of doxazosin. Am J Med 1989; 87: 2S-11S.

29 ALLHAT Officers and Coordinators for the ALLHAT Collaborative Research Group The Antihypertensive Lipid-Lowering Treatment to Prevent Heart Attack Trial. Major outcomes in high-risk hypertensive patients randomized to angiotensin-converting enzyme inhibitor or calcium channel blocker vs diuretic: the antihypertensive and lipid-lowering treatment to prevent heart attack trial (ALLHAT). JAMA 2002; 288: 2981-2997.

30 Chapman N, Chang CL, Dahlof B, Sever PS, Wedel H, Poulter NR. Effect of doxazosin gastrointestinal therapeutic system as third-line antihypertensive therapy on blood pressure and lipids in the Anglo-Scandinavian cardiac outcomes trial. Circulation 2008; 118: 42-48. 